\title{
Quand les mesures d'économie nous bloquent
}

\author{
Josef E. Brandenberg
}

Dr méd., spécialiste en chirurgie orthopédique et traumatologie de l'appareil locomoteur, président de la fmCh

Depuis des années, on nous martèle que le progrès médical est l'une des principales causes de l'augmentation des coûts. Les économies réalisées par les innovations sont occultées. Il existe pourtant bel et bien de nombreux exemples qui montrent que le progrès médical a non seulement amélioré le traitement et la qualité de vie des patients, mais aussi permis des économies.

\section{Comment de nouveaux médicaments ont supplanté les opérations de l'estomac}

A l'examen fédéral en 1976, les futurs médecins devaient savoir répondre aux questions orales et écrites sur la vagotomie. A cette époque, ce traitement chirurgical de l'ulcère gastro-duodénal n'était pas encore obsolète. L'ulcère gastro-duodénal était une maladie fréquente d'une population soi-disant stressée et la vagotomie, le traitement privilégié. Une année plus tard, cette intervention avait disparu des programmes opératoires et était remplacée par la ciméthidine (Tagamet ${ }^{\circledR}$ ). Ce médicament a connu un succès planétaire et est soidisant un des premiers blockbusters [1, 2] ${ }^{1}$ de l'histoire de l'industrie pharmaceutique. Mais personne ne souligne les importantes économies liées à la disparition de la vagotomie.

\section{Comment le monitoring périnatal a réduit les handicaps}

Dans les années 1970, l'obstétrique a fait d'énormes progrès techniques. Soutenu par la recherche clinique et les progrès technologiques, le monitoring des naissances s'est développé à l'aide de la cardiotocographie, de la gazométrie fœtale et de l'électrocardiogramme fœtal obtenu par la pose d'une électrode dans le scalp du fœetus. La détection précoce d'un manque d'oxygène a certes entraîné plus de césariennes, mais a quasiment fait disparaître les lésions cérébrales dues au déroulement de l'accouchement. Grâce au monitoring, on a pu éviter à beaucoup d'enfants des spasmes et d'autres atteintes, et par conséquent aussi des infirmités à vie et les contraintes que cela implique pour les familles. Le dernier cas en date - tragique pour toutes les parties impliquées - montre que l'omission de la surveillance est aujourd'hui considérée comme une violation du devoir de diligence [3]. L'indemnité fixée par le tribunal ne suffit de loin pas à couvrir les coûts consécutifs liés aux traitements médicaux, aux moyens auxiliaires, à l'enseignement spécialisé et aux rentes $\mathrm{AI}$, mais permet d'évaluer quelles économies ont été réalisées grâce à l'obstétrique moderne.

\section{Comment la recherche clinique a évité des opérations aux enfants}

A la fin des années 1970, des enfants occupaient encore des services entiers dans les cliniques, avant tout des jeunes filles prépubères, à qui l'on corrigeait les hanches parce qu'elles marchaient avec les pieds en dedans. La correction s'effectuait avec une ostéotomie intertrochantérienne en sectionnant et tournant l'os, d'abord d'un côté, puis après guérison, de l'autre côté. Des études cliniques - par ex. l'excellente thèse de Schwarzenbach [4] - ont montré que cette antétorsion se corrigeait spontanément jusqu'au terme de la croissance et que le traitement chirurgical ne présentait pas d'avantages [5]. L'ostéotomie de dérotation a très rapidement disparu des programmes opératoires. Une fois de plus, la recherche clinique a permis d'importantes économies.

\section{Autre succès de la recherche: la chaussure stabilisatrice au lieu de la suture ligamentaire}

La distorsion de l'articulation tibio-tarsienne supérieure est la blessure la plus fréquente de la cheville. On a pensé longtemps que les ligaments déchirés devaient 
être suturés. Or, en 1987, une étude randomisée prospective [6] a montré qu'un traitement conservateur apportait d'aussi bons résultats. Dans une autre étude, la supériorité du traitement fonctionnel, sans mise au repos rigide, par rapport à l'opération a même pu être démontrée [7]. Depuis, fabricants et vendeurs de chaussures stabilisatrices font de bonnes affaires. La suture immédiate des ligaments déchirés de l'articulation tibio-tarsienne a disparu des programmes opératoires. Un autre exemple d'économies réalisées grâce à la recherche clinique!

\section{L'échographie de la hanche - l'histoire d'un succès obtenu malgré les obstacles administratifs}

En 1980, le chirurgien orthopédiste Reinhold Graf [8] publia des travaux sur l'ultrasonographie de la hanche chez le nourrisson. Depuis lors, de nombreuses études ont montré que l'ultrasonographique permettait de dépister la dysplasie de la hanche, la malformation la plus fréquente, survenant chez environ $4 \%$ de tous les nouveau-nés. Son traitement précoce réduit ses graves conséquences: de longs séjours hospitaliers pour des très jeunes enfants, des opérations successives, le risque d'arthrose précoce. Grâce à la persévérance de chirurgiens et orthopédistes pédiatriques engagés par ex. Fritz Hefti à Bâle, Ulrich Exner à Zurich, Martin Schilt à Lucerne [9] -, l'échographie de la hanche du nourrisson s'est établie en Suisse et a évité beaucoup de souffrances, mais aussi permis des économies importantes. Incroyable mais vrai: cet examen a failli être retiré du catalogue des prestations de base en 2004 par le Département fédéral de l'intérieur, qui cherchait à économiser à tout va. Il a été maintenu grâce aux protestations du corps médical et avec l'appui de la Commission nationale d'éthique. [10] Aujourd'hui, on n'observe plus les graves conséquences de la dysplasie de la hanche que chez des immigrés, parfois même originaires de pays dont le système de santé est dit supérieur à celui de la Suisse.

\section{La recherche réduit les maladies et révèle les méthodes de traitement inappro- priées, mais doit être financée}

Dr méd. Josef E. Brandenberg Spécialiste en chirurgie orthopédique et traumatologie de l'appareil locomoteur Président de la fmCh Rebstockhalde 18

CH-6006 Lucerne

josef.brandenberg[at]hin.ch niques, mais aussi des méthodes de traitement inappropriées, ce qui permet des économies substantielles. En comparaison, les résultats des mesures d'économie politiques restent modestes.

Malgré tout, le financement de la recherche et du développement devient de plus en plus difficile. Lors d'un podium de l'Arbeitsgemeinschaft für Endoprothetik consacré à la politique de la santé à Berlin en décembre 2016, plusieurs médecins-chefs de cliniques universitaires ont dénoncé le fait qu'il était quasi impossible de réaliser des études cliniques par manque de ressources ou de personnel. Des problèmes semblables apparaissent en Suisse.

L'introduction de la loi sur l'assurance-maladie (LAMal) en 1996 a imposé partout les critères EAE. Les prestations médicales ne sont remboursées par l'assurance obligatoire des soins (AOS) que si leur efficacité, leur adéquation et leur économicité ont été prouvées. Les coûts de recherche et de développement - comme d'ailleurs la formation postgraduée - sont explicitement exclus des prestations de l'assurance sociale. Mais alors, qui doit les prendre en charge?

L'introduction des forfaits par cas dans l'AOS a contraint les hôpitaux à faire encore plus d'économies. Quant à la recherche clinique, elle doit se contenter de recettes issues des assurances complémentaires et de fonds acquis auprès de tiers, alors que le nombre d'assurés complémentaires baisse et que les recettes supplémentaires sont soumises à une pression croissante des autorités de régulation. Enfin, les contributions de l'industrie aux hôpitaux et chercheurs sont volontiers critiquées et impliquent parfois un risque de réputation pour l'hôpital concerné.

\section{Conclusion et résumé}

Les exemples cités de l'opération de l'estomac, de la surveillance de l'accouchement, de l'ostéotomie, des blessures de l'articulation tibio-tarsienne et de la sonographie de la hanche montrent que par le passé, le progrès médical n'a pas seulement entraîné une augmentation des coûts, mais a surtout permis des économies et évité aux patients et à leurs proches beaucoup de souffrances et certains traitements inutiles. Ce genre de progrès s'appuie sur les découvertes réalisées grâce à la recherche clinique, qui sont toujours plus soumises à une pression financière. Les pseudo-mesures d'économie réalisées au détriment de la recherche clinique bloquent pourtant l'innovation, empêchent de véritables économies et rendent le système de santé non pas plus avantageux, mais surtout plus mauvais. 


\section{Références}

1 https://fr.wikipedia.org/wiki/Cim\%C3\%A9tidine

2 https://fr.wikipedia.org/wiki/Blockbuster

3 Tagesanzeiger du 28.10.2017. „Zürcher Spital muss für behindertes Kind Genugtuung zahlen“. Bericht über das Urteil des Zürcher Obergerichts.

4 Schwarzenbach U (1971). Die Rückbildungstendenz der idiopathisch vermehrten Antetorsion des Schenkelhalses. InauguralDissertation. Medizinische Fakultät, Universität Zürich. Arch Orthop Unfallchir;70:230-42.

5 Jani L, Schwarzenbach U, Afifi K, Scholder P, Gisler P (1979). Verlauf der idiopathischen Coxa antetorta. Kontrolluntersuchungen von 148 Patienten bei Wachstumsabschluss. Orthopäde;8:5-11.
6 Korkala O, Rusanen M, Jokipii, Kytömaa J, Avikainen V (1987). A prospektive study of the treatment of severe tears of the lateral ligament of the ankle. Int Orthop;11(1):13-7.

7 Kaikkonen A, Kannus P, Järvinen M (1996).Surgery versus functional treatment in ankle ligament tears. A prospective study. Clin Orthop Relat Res. 1996 May;(326):194-202.

8 Graf R (1980). The diagnosis of congenital hip-joint dislocation by the ultrasonic Combound treatment. Arch Orthop Trauma Surg;97(2):117-33.

9 In memoriam Martin Schilt. Bulletin des médecins suisses 2012;93(11):405

10 Bulletin des médecins suisses 2004;85(12):620. URL : https:// bullmed.ch/article/doi/saez.2004.10368 